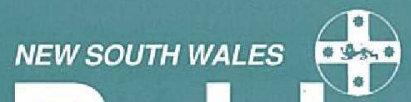 Public

\section{SUICIDE MORTALITY IN NSW LOCAL GOVERNMENT AREAS}

Gavin Stewart, Jennifer Chipps and Geoffrey Sayer

Mental Health Epidemiology,

Epidemiology Branch

Public Health Division, NSW Health Department

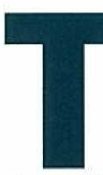

his is the fourth of a series of articles on the epidemiology of suicide in NSW by the Mental Health Epidemiology Group (MHEG). Our first report $^{1}$ presented Standardised Mortality Ratios (SMRs) for Area and District Health Services by pooling data across the 14-year period 197992. In this report we present the same analysis for Local Government Areas (LGAs). This is the finest level of geographic detail available in the Statewide death data.

We demonstrated in our first report that it is not feasible to use the suicide rate as a performance indicator for the success of suicide prevention programs in small populations, such as those of Area and (especially) District Health Services ${ }^{1}$. This applies also to LGAs. However, it is common for questions to be asked about the suicide rate for a particular LGA, and the data here may be useful to those who are called on to answer questions about local suicide rates. We have grouped LGAs under the relevant Area or District Health Service and repeated the previous data for those Areas and Districts. This helps to indicate ways in which the data may be used to inform local discussions of suicide and prevention activity.

\section{METHOD}

Australian Bureau of Statistics suicide mortality data for Area and District Health Services and LGAs were pooled over the period 1979-92 and indirectly standardised relative to the pooled data for NSW during the same period. SMRs were scaled in the conventional way so SMR=100 for NSW as a whole. This standardisation adjusts for differences in the age-sex composition of the pooled population of the particular geographic area relative to the pooled NSW population. Thus an SMR of 120 means the particular area had a pooled suicide rate which was 20 per cent higher than that for NSW as a whole over the same period. Conventional 99 per cent confidence intervals were calculated for each SMR based on the usual assumption of Poisson-distributed events.

As some boundaries between LGAs have changed over time, we used aggregate data for LGAs where this was the case ${ }^{2}$. Data for the LGAs of Central Sydney and Southern Sydney, Blacktown and Parramatta, Warringah and Pittwater, and a number of rural LGAs are presented for these groups, rather than for individual LGAs.

Most health administrative boundaries in NSW follow LGA boundaries. In general, therefore, it is possible to add up the observed and expected deaths for the set of LGAs reported under an Area/District heading, and arrive at the total for that Area or District. The exceptions to this are the composite LGA of Sydney and Southern Sydney (which divides in the proportion 40:60 between Central Sydney AHS and Eastern Sydney $\mathrm{AHS}^{3}$ ) and the LGA of Blayney (which is part of the composite LGA of Orange/Blayney/Cabonne because of boundary changes among these LGAs over time). We have reported data for Orange/Blayney/Cabonne under the Central Western DHS, but part of it belongs to the Evans DHS. Thus the data

\section{Contents}

\section{Articles}

1 Suicide mortality in NSW Local Government Areas

11 Waiting List Reduction Program, March to

December 1995

\section{Infectious Diseases}

\section{Notification trends}

\section{Correspondence}

Please address all correspondence and potential contributions to:

\section{The Editor,}

NSW Public Health Bulletin, Public Health Division, NSW Health Department Locked Bag No 961 , Nortb Sydney NSW 2059 Telephone: (O2) 3919191 Facsimile: (O2) 3919029 


\section{Suicide mortality in NSW}

\section{Continued from page 1}

for each of the Areas and Districts are correct, and the data for individual LGAs and composites are correct, but the individual LGAs for Eastern and Central Sydney have to be combined to match the total deaths in those two areas. The same applies to Central Western and Evans DHS.

Recent changes in NSW Health administrative boundaries are only partly reflected in these data. We have reported the data for Canterbury LGA under both Southern Sydney AHS (where it forms part of the total for the AHS), and under Central Sydney AHS (where it now belongs, but does not, in this data, contribute to the AHS total). We have grouped Districts according to the Public Health Units which serve them, as in the previous report.

\section{RESULTS}

In each of Figures 1-6, the name of the Area or District or LGA is followed by the number of actual deaths by suicide over the 14-year period 1979-92, then the expected number of deaths for that locality if the NSW average age-specific death rates had applied to that population during the same period. The latter have been rounded to the nearest whole number.

Thus at the top of Figure 1 we show Central Sydney AHS with 737 actual deaths as against 570 which would have been expected for that population on State average rates. Immediately after the data for Central Sydney AHS are the data for the composite of Southern Sydney LGA and Central Sydney LGA: 328 actual deaths and 154 expected at State average rates. Of these, 40 per cent contribute to the totals for Central Sydney AHS. Data for LGAs are reported in descending order of SMRs within the Area or District.

For localities with at least five deaths, the graph shows the SMR and its 99 per cent confidence interval (CI). In the case of a few LGAs with very small populations, the value of the upper 99 per cent confidence limit exceeds SMR=300 and is not shown. The shaded area is the 'target' area of a 10 per cent reduction in the average suicide mortality for the State as a whole.

\section{DISCUSSION}

The data for 151 individual LGAs demonstrate, as previously, that estimates of the rates of rare events are very imprecise in small populations. There are various technical issues associated with the interpretation of these results, some of which were addressed in our first paper ${ }^{1}$, and in correspondence arising out of $\mathrm{it}^{4}$. One of the agreed conclusions from that debate is that there is a need to offer guidance in the analysis and interpretation of health data of this kind.

For those reasons, we have added an account of some of the issues that arise when a large body of data is explored to detect "unusual" variation. The technicalities have been placed in footnotes as far as possible, and the main focus of the discussion is on the practical usage of the results.

\section{Assessment of overall variation of LGAs relative} to the NSW average

It is technically incorrect to compare indirectly standardised rates or SMRs with one another unless relatively sophisticated procedures are used ${ }^{5,6}$ and we have therefore interpreted each SMR in relation to $\mathrm{SMR}=100$ for NSW as a whole. As a practical decision rule, we used the 99 per cent CI surrounding the SMR estimate for an LGA to classify the LGA as "unusually" high or low relative to $S M R=100^{7}$. Thus if SMR=100 lies below the lower 99 per cent CI bound for the SMR of the LGA, we consider that LGA as having an unusually high average suicide rate (for example, Marrickville, in Figure 1). Similarly, if SMR=100 lies above the upper 99 per cent CI bound for the SMR of the LGA, we consider that LGA as having an unusually low average suicide rate (for example, Hornsby, in Figure 1).

Using these definitions, there are eight LGAs with unusually high SMRs for suicide: Southern/Central Sydney, Leichhardt, Marrickville, Woollahra, Waverley, Randwick and North Sydney (Figure 1) and Broken Hill (Figure 4). There are also 10 LGAs with unusually low SMRs for suicide: Canterbury, Sutherland, Hornsby, Ku-ring-gai and Ryde (Figure 1), and Fairfield, Campbelltown, Baulkham Hills, Lake Macquarie and Port Stephens (Figure 2).

Clearly, there are other LGAs in Figures 1-6 which are "almost unusual", and not all the unusual LGAs are equally so. The confidence intervals shown in Figures 1-6 provide a much more informative view of these data than the simple classification based on an hypothesis-testing approach ${ }^{8}$. Nevertheless, in practice, confidence intervals tend to be used to classify groups of data, more or less as outlined above. It is therefore useful to estimate the probable errors involved in making decisions about geographical or administrative units in this way.

In classifying LGAs as unusually high or low, we concluded either that the result SMR=100 is within the range of likely values for the suicide rate in the LGA (negative decision not "unusual"), or that it is not (positive decision "unusual"). Because the test is based on a 99 per cent CI, we know there is a 1 per cent false positive rate for each individual decision. We made 151 individual decisions: one per LGA, and we can therefore expect that one or two "false positives" will result 9 . We can also calculate that the chance of obtaining a total of 18 "false positives" is essentially zero $^{10}$. Taken together, these results mean we can safely conclude that there is some systematic variation in the standardised suicide mortality between LGAs in NSW, but we should not be preoccupied with the difference between the 18 "positives" (since one or two are likely to be false positives) and the remaining 133 LGAs.

Another useful way of looking at the data is to classify LGAs in relation to some meaningful SMR value other than SMR=100. We might, for example, accept as "less problematic" any LGA whose suicide rate can confidently be said to be no more than 20 per cent above the State average. These are the LGAs for which SMR=120 lies above the upper 99 per cent CI bound. This would add the LGAs of Botany, Hurstville, Kogarah, Warringah, Lane Cove (Figure 1), Bankstown, Camden, Wollondilly, Parramatta/Blacktown, Penrith, Hawkesbury, Newcastle, Wollongong, Shoalhaven (Figure 2), and Hastings (Figure 3) to the 10 LGAs with unusually low average suicide rates.

However, the most useful way of expressing the result for any particular LGA is in terms of its confidence interval. The most we can actually say is that, for example, the average suicide rate in Penrith LGA over the period 1979-92 was about 90 per cent of the State average, and there is a 99 per cent chance that the true value is between 75 per cent and 110 per cent of the State average. Data of this kind can be read off Figures 1-6 for every LGA in NSW. 


\section{Assessment of variation within health administrative areas}

Most of the LGA data simply add detail to our previous conclusions about Area Health Services and Districts. The Central Sydney and Eastern Sydney Areas were classified as having unusually high SMRs in our previous analysis, and this is true of most (but not all) of the LGAs within them (Figure 1). The Far West District also has a high SMR, and this is clearly due to the LGA of Broken Hill (Figure 4). The Southern Sydney Area (Figure 1) and South Western Sydney Area (Figure 2) had unusually low SMRs in our previous analyses, and this is true of some of the LGAs within them.

The main value of the LGA-level analyses therefore lies in providing an indication to Area Health Services (and, to a lesser extent, District Health Services) of within-area variation. Looking at the variation in SMRs across LGAs in the same area gives an indication of whether the problem seems to be localised or uniform. For example, the Northern Sydney AHS as a whole is not different from the State average, but it contains an LGA (North Sydney) with one of the high rates and three LGAs (Hornsby, Ku-ring-gai, Ryde) with low rates.

The newly created South Eastern Sydney AHS will have an overall SMR closer to the State average than that of either of the original AHSs, because it will include four of the eight LGAs with high suicide rates (all from Eastern Sydney), and two of the 10 LGAs with low suicide rates (bot' 'rom Southern Sydney). Clearly, the suicide problem is not solved by administrative mergers of this kind, but at the level of statistical summaries it might appear to be. For that reason alone it is useful to report data at a finer level of geographical detail. Similarly, the addition of Canterbury to Central Sydney AHS will reduce the suicide rate for the new AHS as a whole as the result of "immigration" of a lowerrate population, just as the "emigration" of that population from Southern Sydney AHS would have raised the average for the new area.

A practical way of looking at this is to consider the estimated SMRs for the LGAs within an Area or District against the 99 per cent CI for the Area or District as a whole. This violates the rule about not comparing indirectly standardised SMRs with one another ${ }^{5}$, but is a reasonably valid way of assessing the uniformity of the suicide rate across geographical subdivisions of a larger area. For example, in the Northern Sydney AHS (Figure 1), there are four LGAs (Hunters Hill, Manly, North Sydney, Mosman) whose SMRs lie above the upper 99 per cent confidence limit for the AHS as a whole, and another four (Hornsby, Lane Cove, Ku-ring-gai, Ryde) whose SMRs lie below the lower limit. On this basis, those concerned with suicide prevention in the Northern Sydney AHS might choose to focus on the former group of LGAs. By contrast, the data for the Wentworth, Central Coast and Illawarra Area Health Services (Figure 2) show quite uniform SMRs across the relevant LGAs, and an Area-wide approach might be more suitable.

It may also be noted that the LGAs with the highest SMRs in the Northern Sydney AHS would be regarded as "average" in Central Sydney AHS and are below the average in Eastern Sydney AHS, but issues of this kind are better dealt with by considering the Area or District Health Service as a whole in relation to the NSW average.

\section{Measures to set and monitor progress} towards targets

A number of useful measures may also be derived from the data to assist local discussions on suicide issues, and in planning how to set and monitor progress towards targets. These measures are of most value to District Health Services where populations are small, annual data are very variable and sophisticated statistical advice may be difficult to obtain. We will illustrate some uses of the reported data by referring to the Murrumbidgee DHS (Figure 5).

Next to each locality in Figures 1-6 we have presented the number of actual deaths, and the number of deaths expected if the NSW average age-specific suicide rates applied through the years 1979-92. The ratio of these (actual/expected), expressed as a percentage, is the SMR. Thus for the Murrumbidgee DHS the SMR can be calculated ${ }^{11}$ as (SMR=actual deaths as percentage of expected deaths $=79 / 76 \times 100=104$ ). The plotted point scaled off Figure 5 is about 105.

It is also useful to present the same evidence as "excess mortality". For the Murrumbidgee DHS as a whole there would have been 76 deaths by suicide in 14 years if people in that District were at the same risk as others in the State as a whole. In fact there were 79 deaths, so the excess mortality of the District due to suicide amounted to three deaths in 14 years. This was not uniform across the District, however, since the individual LGA data show there were four excess deaths in Hay, five in Leeton and one in Narrandera, while Griffith had six fewer deaths than expected, Carrathool one fewer and Murrumbidgee had the same suicide mortality as the rest of the State. To avoid differences of this kind being over-emphasised, it should be noted that "excess mortality" is the difference between actual and expected deaths, while an SMR is the ratio of actual to expected deaths, expressed as a percentage, which is equal to 100 when actual deaths equals expected deaths. Therefore, since the SMRs for all the individual LGAs in the Murrumbidgee DHS, and for the DHS as a whole, have wide confidence intervals which include $S M R=100$, it follows that the corresponding confidence intervals for the excess mortality are wide, and include excess mortality $=\mathrm{zero}^{12}$.

Similar calculations can be used to determine the expected number of deaths for other SMR values. Suppose the District administration chose to aim at a reduction to $\mathrm{SMR}=90$, as indicated in the target range. What actual number of deaths would correspond to $\mathrm{SMR}=90$ ? This can be determined in the following way. SMR=100 corresponds to the expected number of deaths (76). Therefore SMR $=90$ corresponds to 90 per cent of the expected number of deaths $(90 / 100 \times 76=68.4)$. This is the number of expected deaths over 14 years. This corresponds to a change from $79 / 14=5.6$ per year to $68.4 / 14=4.9$ per year. For the Murrumbidgee DHS, therefore, a target of SMR $=90$ relative to the NSW average rate between $1979-92$ corresponds to $5.6-4.9=0.7$ deaths a year. This target (one life saved a year) is easier to relate to than achieving a reduction in the SMR from 105 to 90 . It also may help to consider the relative benefit of investments in this area of health care as against other life-saving interventions.

Calculations of this kind can readily be carried through for any SMR that is chosen as a target, for any of the data in Figures 1-6. It must be remembered, though, that these are 14-year data for the period 1979-92, so even though the data 


\section{Suicide mortality in NSW}

\section{- Continued from page 3}

are more reliable, they are not very sensitive to recent population shifts or changes.

\section{CONCLUSIONS}

The main value of the LGA-level analyses lies in providing indications to those concerned with suicide prevention in Area and District Health Services. This may help in explaining the issues at the local level, in targeting programs on smaller administrative areas which have higher SMRs than others, and perhaps in developing programs with local government. In addition, LGA-level data are useful in avoiding the possibility that health problems which are distributed unevenly in the population can become invisible when various sub-populations are merged and only aggregate data are reported. This is well illustrated by the recent merging of the former Eastern Sydney and Southern Sydney Area Health Services (less Canterbury LGA) to create the South-Eastern Sydney AHS. There is, therefore, a clear role for Public Health Units in addressing these issues of within-area variation in health status indicators.

There is also a value in understanding the statistical basis of interpreting observed data. The suicide risk for a population results from a multiplicity of factors - some chronic, some acute, some affecting the population as a whole, and some affecting only a few individuals within it. In a fairly typical LGA in NSW, with a population of 20,000 males, constant over the period 1979-92, there were four male deaths a year on average, but in some years as many as eight and in one year no deaths at all. The only "pattern" in the data which would allow the number in any particular year to be predicted is that it was consistent with a Poisson distribution with a mean of four deaths a year. If we take this as a model, we know there is only about one chance in 100 of 10 or more deaths occurring if the underlying risk has remained constant. Between the unobservable risk, and the observable data, lie all the unknown factors which affect the individuals in a population. The power of a statistical model is that it makes efficient use of all the available information to guide decisions and to provide indicators against which we can measure suicide prevention programs. In particular, it gives us an estimate of the extent to which the observed data will vary even when the underlying risk remains the same.

By contrast, if we do not take this into account when we assess the performance of suicide prevention programs against observable indicators, the decisions we make on programs may be as random as the year-to-year variation in suicide deaths in a typical LGA in NSW. The alternative is not to abandon the attempt to address these problems on a local level, but to choose appropriate indicators of performance. Programs which have a logical relationship to risk reduction in the population, and which are agreed by relevant expert opinion - including the opinion of community groups - to be of value, need to be implemented and sustained for long enough to have an effect which is detectable. Every reasonable effort must be made to establish a connection between program activity and its impact on risk, even if it is impossible to carry the argument through to a demonstrable impact on the suicide rate.

\section{ACKNOWLEDGMENTS}

We wish to thank Dr Tim Churches, Epidemiology Branch, NSW Health Department, for supplying the SAS routine which was adapted for the standardisation, and Kerrie Crawford and Margaret Riakos from the Centre for Mental Health for verifying the data in the figures.

\section{NOTE}

Membership of MHEG is open to people with a professional interest and expertise in mental health epidemiology who are willing to contribute to the planning and production of a series of publications and reports on important mental health topics. The policy of MHEG is joint publication by the group as a whole in which authors are listed in order of their contribution to the particular report. The contact address for matters concerning this report is Mental Health Epidemiology Group, Centre for Clinical Policy \& Practice, Public Health Division, NSW Health Department, Locked Bag 961 PO North Sydney 2059 (Fax: 391-9232, Internet e-mail gstew@gwsm.doh.health.nsw.gov.au).

1. Stewart G, Chipps JA, Sayer G. Suicide mortality in NSW: geographic variations. NSW Public Health Bulletin, 1995; 6(6):49-52.

2. The mortality and population data used in this report contain codes for "continuous" LGAs. These are either individual LGAs whose boundaries have not changed over the period 1970-1995, or combinations of LGAs in cases where the inter-LGA boundary has changed, but the boundary for the aggregate has not. These data were prepared for the HOIST (Health Outcomes Information and Statistical Toolkit) system by Dr Tim Churches and his group, based on similar files developed by the NSW Central Cancer Registry.

3. This apportionment has been done by a particular randomised allocation of deaths in the mortality file in the HOIST data system, since the place of residence of the person at death is only classified to the LGA On average the assignment is in the ratio 40:60, but in any year or group of years it will not be exactly $40: 60$ because of the randomisation. This problem arises only because of the arbitrary boundary chosen to divide these LGAs for NSW Health administrative purposes.

4. The discussion can be found in the NSW Public Health Bulletin, 1995; 6(9):92-94. The essential point is that our confidence intervals may, in some circumstances, be underestimates of the true width of the 99 per cent confidence intervals.

5. Carriere KC and Roos L. Comparing standardised rates of events. Am J Epidemiol 1994; 14:472-482.

6. Chan LY, Gibberd RW. Statistical properties of an index to measure systematic regional variation in mortality. Unpublished paper, October 15,1991 .

7. Technically, we are not classifying the result for the LGA as "high" or "low", but rather concluding either that the result SMR=100 was within the range of likely values for the suicide rate in the LGA, or it was not. 8. Gardner MJ, Altman DG (eds). Statistics with confidence. London: British Medical Journal, 1992.

9. If there is a 1 per cent chance of a false positive for each test, and 151 tests, the expected number of false postitives is 1.51, so "one or two" is a practical summary of this result. There is also a false negative rate, which would need to be based on some chosen level of SMR judged "important to detect". For example, if it were considered important to detect SMR=200 (that is, that the suicide rate in a geographical area was twice the State average), it would be possible to estimate the false negative rate for each area. This would be different for each, and depend on the population, but clearly the false negative rates will be high because the confidence intervals at the LGA level are so wide. Even when the true rate was equivalent to $S M R=200$, many of the confidence intervals would still include $S M R=100$.

10 . If the chance of a single false positive is 0.01 ( 1 per cent), then the binomial probability of obtaining more than 12 false positives in a series of 151 tests is less than $10^{-s}$.

11. For those who may wish to report the SMR at the highest accuracy available, greater precision will be obtained by scaling off the point plotted in the relevant figure for small numbers of deaths (because the figure given for expected deaths has been rounded to the nearest whole number) and will be obtained by calculating from the actual and expected death data otherwise.

12. If we cannot conclude that the SMR for the District differs from $\mathrm{SMR}=100$, then neither can we conclude that excess mortality differs from zero. 
FIGURE 1

LOCALITY, OBSERVED DEATHS/NSW AVERAGE AND SMRS FOR SUICIDE PERSONS NSW 1979-92

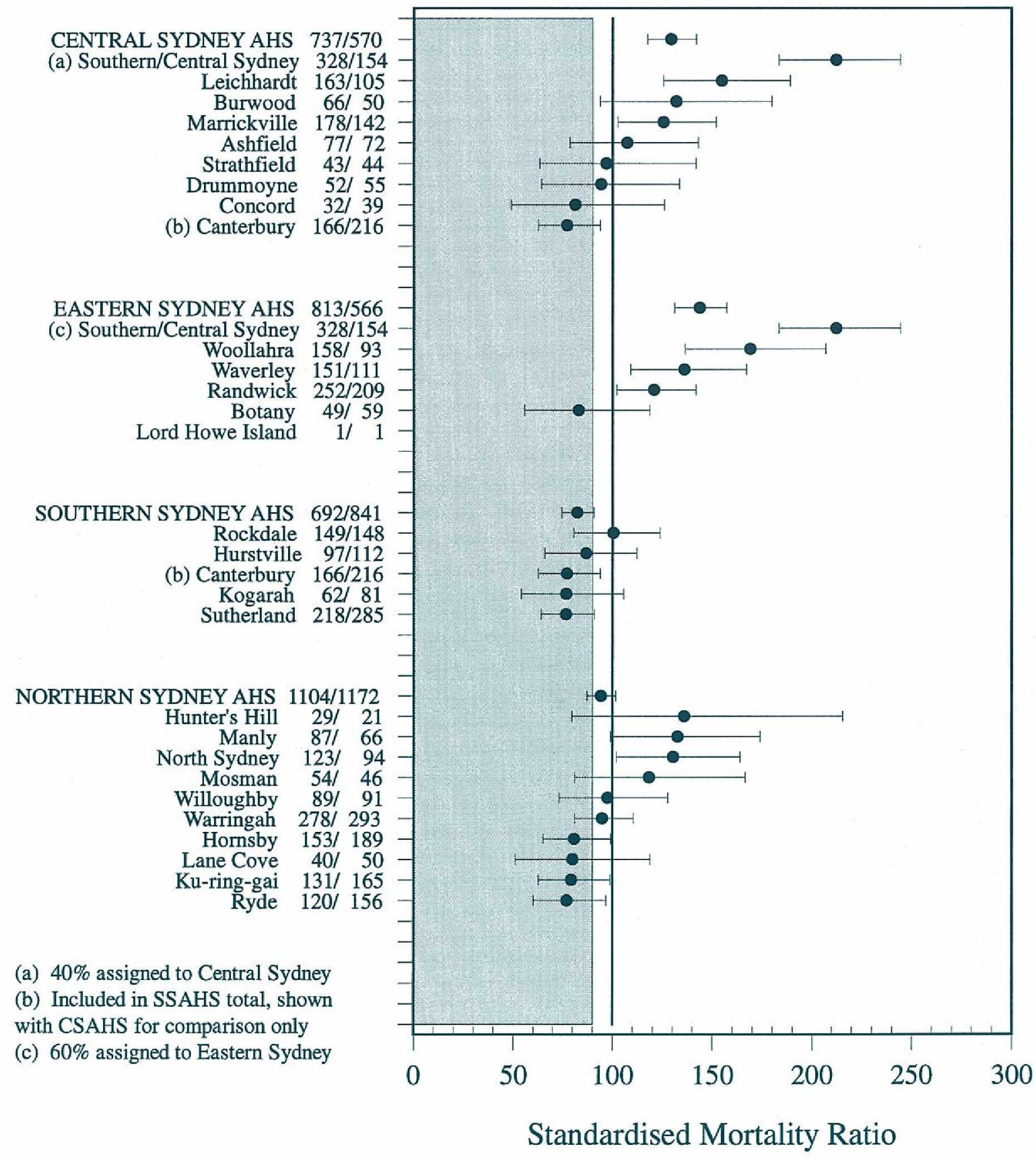

\footnotetext{
I $99 \%$ confidence interval/Poisson

- SMR $=100$ for NSW $1979-92$

SMR for Area/District of residence 1979-92

Target SMR range $<=90$
}

Source: Epidemiology Branch, ABS deaths 1979-92, registered by 1993 


\section{FIGURE 2}

LOCALTTY, OBSERVED DEATHS/NSW AVERAGE AND SMRS FOR SUICIDE PERSONS NSW 1979-92

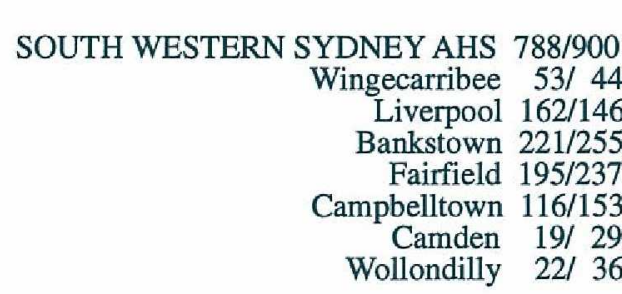

WESTERN SYDNEY AHS 801/857

Parramatta \& Blacktown 502/498

Holroyd 126/128

Auburn 75/ 79

Baulkham Hills 98/153

WENTWORTH AHS 313/349

Blue Mountains 88/96

Penrith 171/188

Hawkesbury 54/ 66

CENTRAL COAST AHS 338/316

Wyong $149 / 137$

Gosford 189/179

HUNTER AHS 674/745

Murrurundi 6/ 4

Merriwa 6/ 4

Maitland 72/ 66

Muswellbrook 22/ 22

Cessnock 64/ 65

Newcastle 227/231

Scone $12 / 14$

Singleton 21/ 25

Lake Macquarie 201/247

Dungog 7/ 11

Port Stephens 36/56

ILLAWARRA AHS 426/462

Shellharbour $70 / 66$

Kiama 15/ 22

Wollongong $260 / 282$

Shoalhaven $81 / 92$

(1/ 92

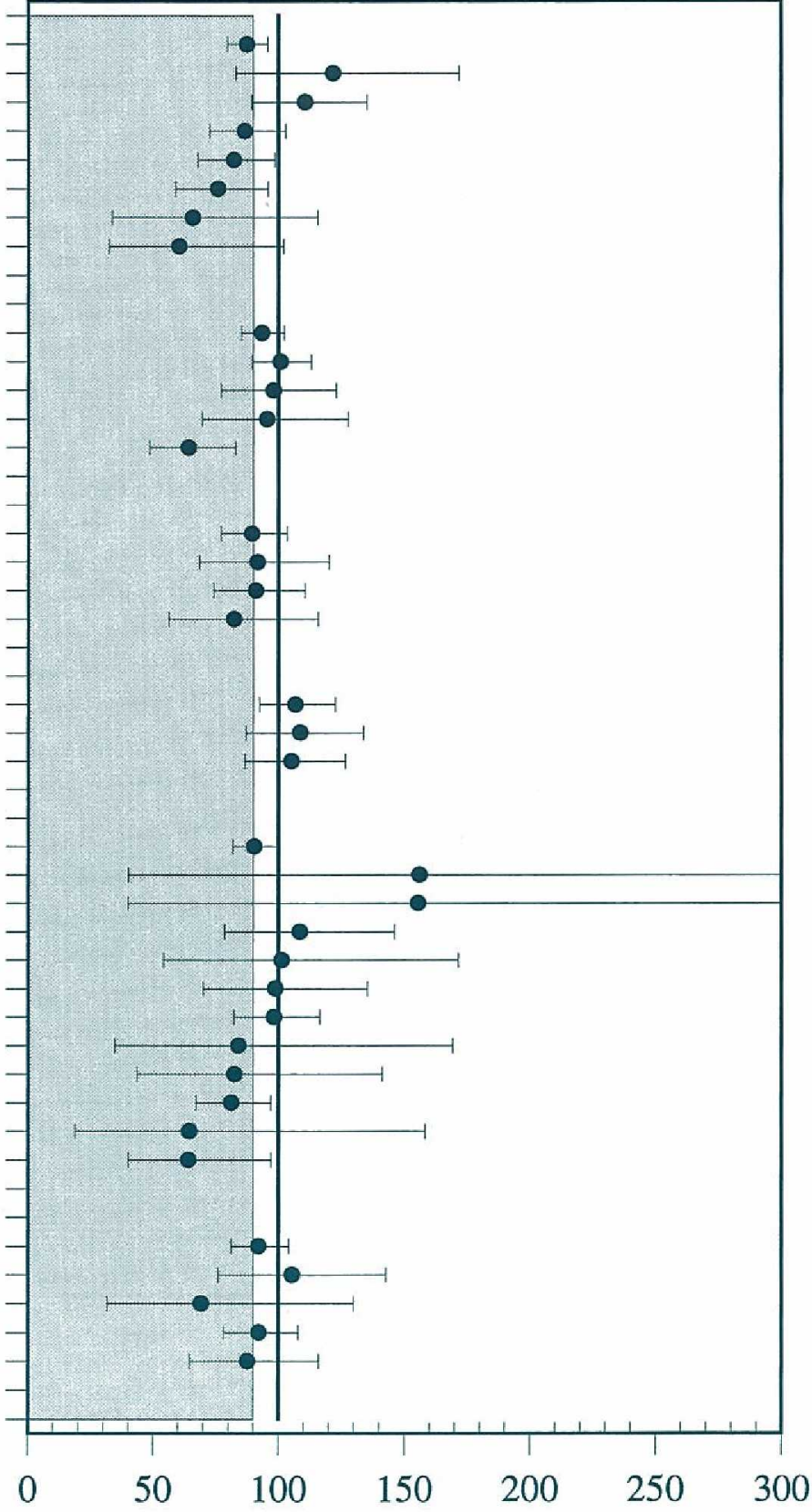

\section{Standardised Mortality Ratio}

I $99 \%$ confidence interval/Poisson

SMR $=100$ for NSW 1979-92

SMR for Area/District of residence 1979-92

Target SMR range $<=90$

Source: Epidemiology Branch, ABS deaths 1979-92, registered by 1993 
FIGURE 3

LOCALITY, OBSERVED DEATHS/NSW AVERAGE AND SMRS FOR SUICIDE PERSONS NSW 1979-92

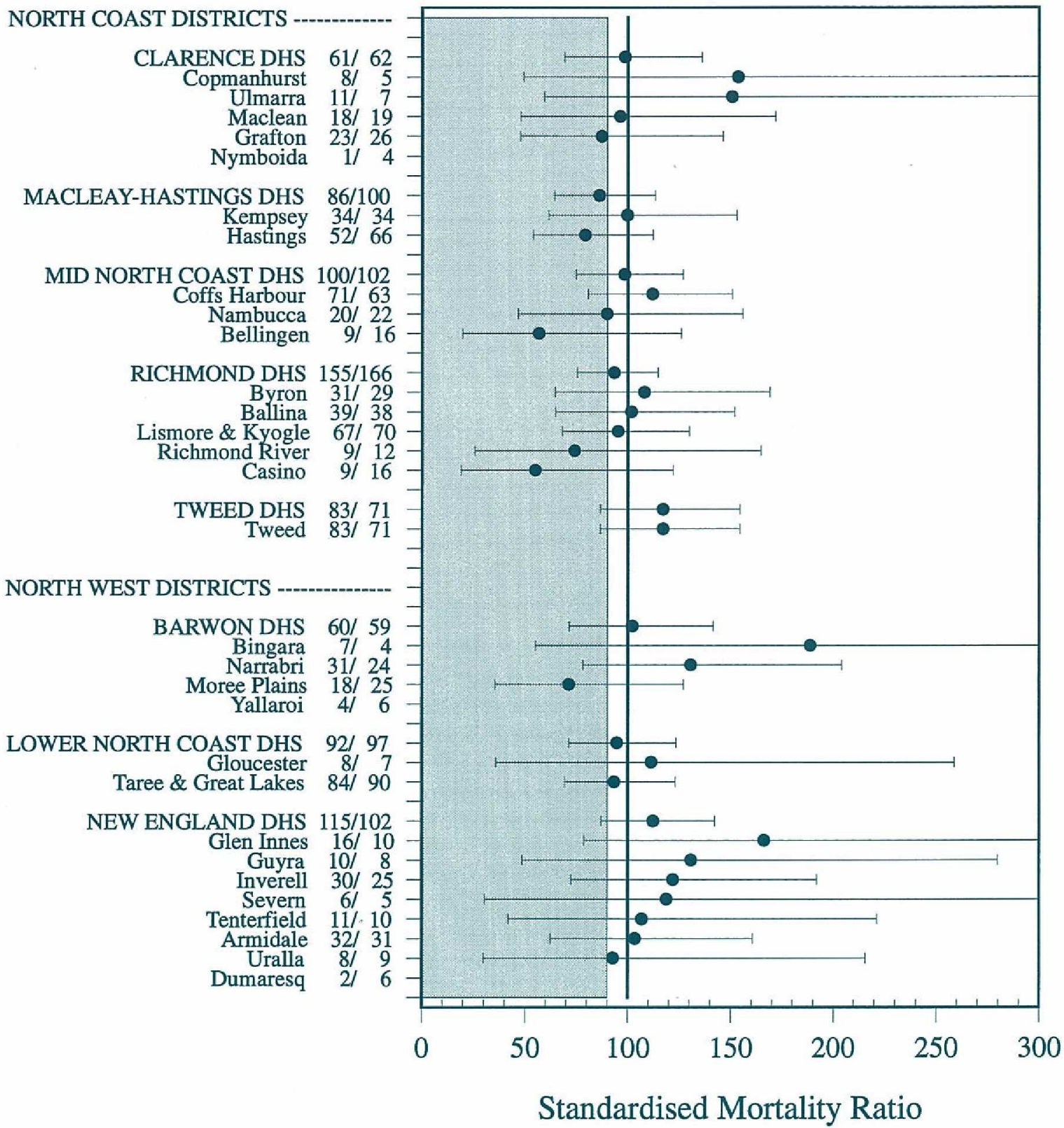

I $99 \%$ confidence interval/Poisson

- $\mathrm{SMR}=100$ for NSW $1979-92$

SMR for Area/District of residence 1979-92

Target SMR range $<=90$

Source: Epidemiology Branch, ABS deaths 1979-92, registered by 1993 
FIGURE 4

LOCALITY, OBSERVED DEATHS/NSW AVERAGE AND SMRS FOR SUICIDE PERSONS NSW 1979-92

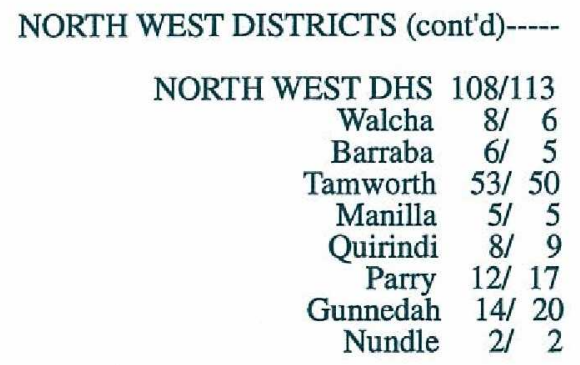

WESTERN DISTRICTS -

CASTLEREAGH DHS

Mudgee

Coonabarabran

Coolah

FAR WEST DHS $95 / 48$

Broken Hill $86 / 41$

Central Darling $7 / 5$

Unincorp Far West 2/ 2

MACOUARIE DHS $120 / 105$

Cobar 15/ 8

Warren 9/ 6

Dubbo 55/ 45

Wellington $17 / 14$

Bogan 6/6

Coonamble $7 / 9$

Gilgandra $6 / 8$

Narromine 5/10

ORANADHS $27 / 22$

Walgett $16 / 12$

$\begin{array}{rrr}\text { Bourke } & 8 / & 7 \\ \text { Brewarrina } & 3 / & 4\end{array}$

CENTRAL WEST DISTRICTS

CENTRAL WESTERN DHS 94/ 91

Cowra 19/18

$\begin{array}{ccc}\text { Orange \& Blayney \& Cabonne } & 70 / 76 \\ \text { Weddin } & 5 / 7\end{array}$

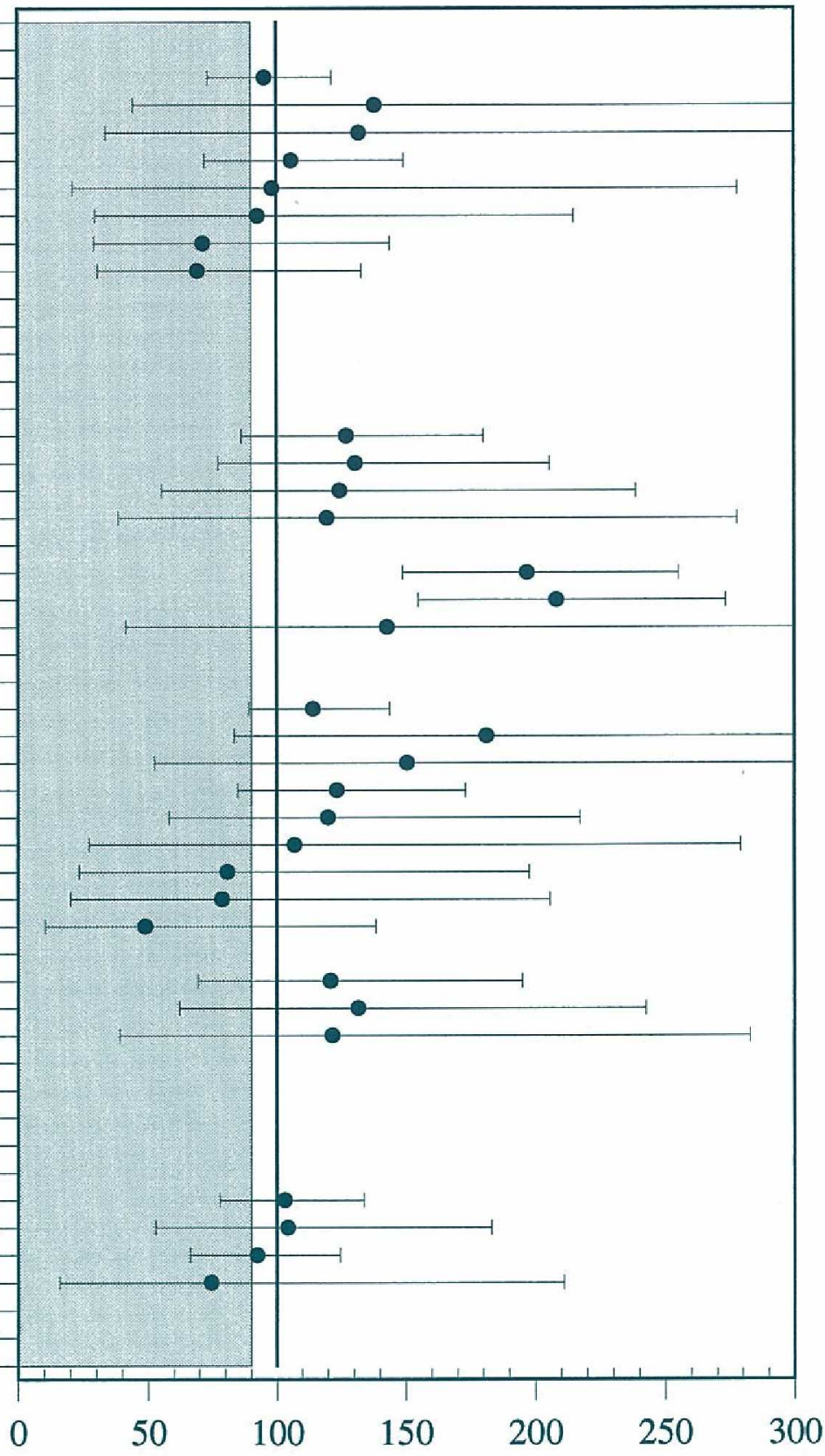

Standardised Mortality Ratio

I $99 \%$ confidence interval/Poisson

SMR for Area/District of residence 1979-92

- SMR $=100$ for NSW 1979-92

Target SMR range $<=90$

Source: Epidemiology Branch, ABS deaths 1979-92, registered by 1993 


\section{FIGURE 5}

LOCALITY, OBSERVED DEATHS/NSW AVERAGE AND SMRS FOR SUICIDE PERSONS NSW 1979-92

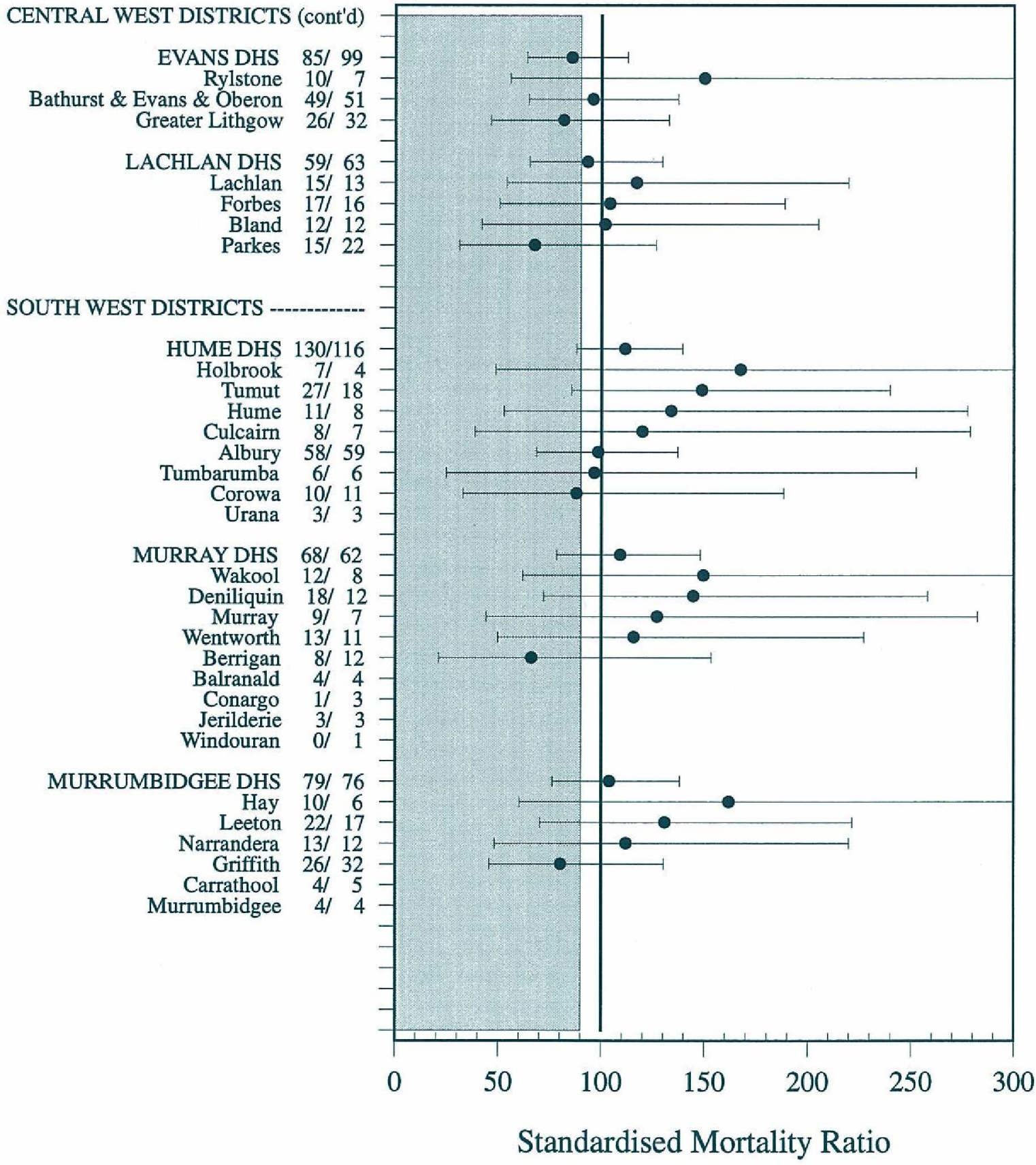

I $99 \%$ confidence interval/Poisson

- $\mathrm{SMR}=100$ for NSW 1979-92

SMR for Area/District of residence 1979-92

Target SMR range $<=90$

Source: Epidemiology Branch, ABS deaths 1979-92, registered by 1993 
FIGURE 6

LOCALITY, OBSERVED DEATHS/NSW AVERAGE AND SMRS FOR SUICIDE PERSONS NSW 1979-92

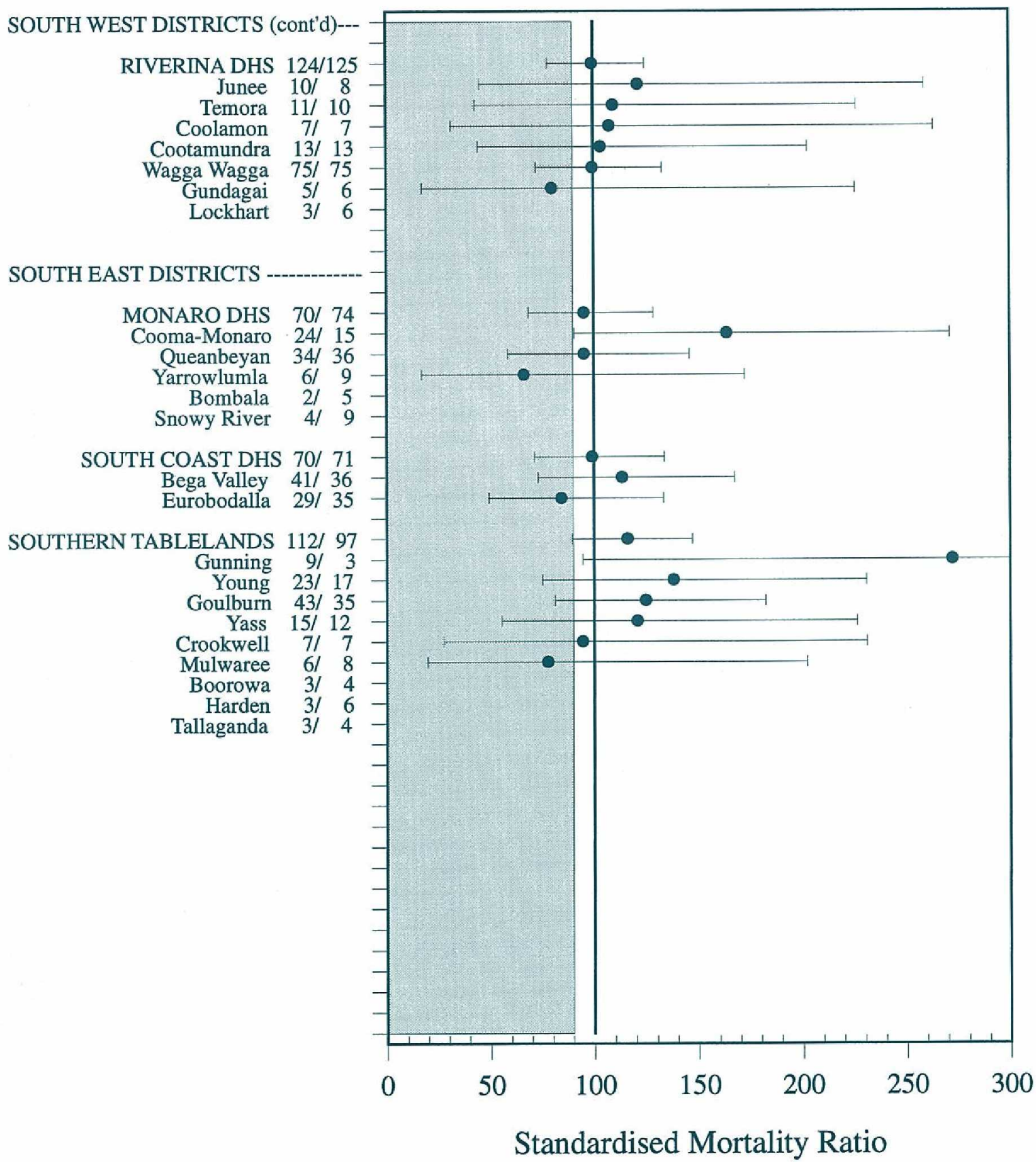

I $99 \%$ confidence interval/Poisson

SMR for Area/District of residence 1979-92

- SMR = 100 for NSW 1979-92

Target SMR range $<=90$

Source: Epidemiology Branch, ABS deaths 1979-92, registered by 1993 